\title{
Introducing Multimodal Paper-Digital Interfaces for Speech-Language Therapy
}

\author{
Anne Marie Piper, Nadir Weibel, James D. Hollan \\ Distributed Cognition and Human-Computer Interaction Lab \\ Department of Cognitive Science, University of California, San Diego \\ 9500 Gilman Dr., La Jolla, CA 92093-0515 \\ \{apiper,weibel,hollan\}@ucsd.edu
}

\begin{abstract}
After a stroke or brain injury, it may be more difficult to understand language and communicate with others. Speechlanguage therapy may help an individual regain language and cope with changes in their communication abilities. Our research examines the process of speech-language therapy with an emphasis on the practices of therapists working with adults with aphasia and apraxia of speech. This paper presents findings from field work undertaken to inform the design of a mixed paper-digital interface prototype using multimodal digital pens. We describe and analyze therapists' initial reactions to the system and present two case studies of use by older adults undergoing speech-language therapy. We discuss the utility of multimodal paper-digital interfaces to assist therapy and describe our vision of a system to help therapists independently create custom interactive paper materials for their clients.
\end{abstract}

\section{Categories and Subject Descriptors}

H.5 [Information Interfaces and Presentation]: User Interfaces

\section{General Terms}

Design, human factors

\section{Keywords}

Communication, speech-language therapy, older adults, multimodal interaction, pen-based computing

\section{INTRODUCTION}

According to the National Stroke Association, stroke is the third leading cause of death in America and a leading cause of adult disability ${ }^{1}$. A stroke or cerebrovascular accident (CVA) occurs when an artery of the brain becomes clogged or ruptured and disrupts the blood flow to part of the brain.

\footnotetext{
${ }^{1}$ http://www.stroke.org
}

Permission to make digital or hard copies of all or part of this work for personal or classroom use is granted without fee provided that copies are not made or distributed for profit or commercial advantage and that copies bear this notice and the full citation on the first page. To copy otherwise, to republish, to post on servers or to redistribute to lists, requires prior specific permission and/or a fee.

ASSETS'10, October 25-27, 2010, Orlando, Florida, USA.

Copyright 2010 ACM 978-1-60558-881-0/10/10 ...\$10.00.
Brain tissue can die or be injured causing a change in neurological function. Strokes occurring on the left side of the brain often result in loss of speech and language, commonly diagnosed as aphasia. The National Aphasia Association reports that of the estimated 400,000 strokes which occur each year, 80,000 result in aphasia ${ }^{2}$. These individuals may understand written and spoken language but be unable to speak fluently or write. A stroke on the left side of the brain may also impair right side motor movement, including arm, hand, and leg functioning.

In addition to aphasia, other speech and language challenges may become present due to a stroke, traumatic brain injury (TBI), or neurological condition. For example, aphasia is often accompanied by apraxia of speech, where an individual is unable to create the voluntary mouth and tongue movements necessary for pronouncing words correctly. In addition, individuals may be unable to translate sounds into meaningful language or separate speech from background noise, also called auditory overload. Slurred speech (dysarthria) and swallowing problems (dysphagia) are other common challenges for this population.

The process of speech-language therapy is designed to help individuals with communication challenges regain their ability to speak, read, write, and understand spoken language. In this work we examine this process, explore how digital pen and paper technology might support it, and introduce a multimodal paper-digital system into this setting.

\section{RELATED WORK}

A range of augmentative and alternative communication (AAC) devices exist for supporting individuals during speechlanguage therapy and in their daily lives. Technologies may be augmentative (support verbal speech and language) and/or alternative (take the place of verbal speech and language). Across the literature on AAC devices, the majority of systems provide multimodal representations of language through written text, speech or sounds, and images. Popular commercial devices include Dynavox ${ }^{3}$ and Lingraphica ${ }^{4}$, both of which are high-tech picture-based systems with speech generators.

Research-based prototypes also exist to support the needs of people with communication challenges, primarily individuals with aphasia. PhotoTalk, for example, is designed to help individuals with aphasia capture and manage digital

\footnotetext{
${ }^{2}$ http: //www. aphasia.org

${ }^{3}$ http : //www.dynavoxtech.com

${ }^{4}$ http: //www. aphasia.com
} 
photos that facilitate face-to-face communication [1]. Moffatt et al. [6] designed a sound and image enhanced daily planner to support individuals with aphasia. Chandler et al. [3] created a mobile web application to support the process of word finding (or word searches) that individuals with aphasia often experience. In addition to helping individuals communicate more effectively and efficiently, some systems are designed to help users perform a task, such as cooking [11] or browsing the Internet [4], more independently.

Few systems, however, serve as a toolkit to aid therapists working with individuals with communication challenges. Bungalow Software ${ }^{5}$ is an example commercial product that allows therapists to tailor computer-based speech and language activities to clients' needs, but it is limited to a computer workstation with activities following a predefined script. Another example is SpeechKit, a multimedia system for use by speech therapists to help rehabilitate individuals with motor impaired communication [2]. More broadly, there is a need to understand the practices of speech-language therapists in order to design tools to better support therapy activities.

The vast majority of HCI research has focused on purely digital AAC devices, yet this neglects the importance of paper-based practices and potential of digital pen technology [7]. Myriad free or inexpensive paper-based resources are available to support speech-language therapy. These include, for example, paper activity worksheets, picture books, and communication boards that allow clients to point to various icons or text as a means of communication. It has been demonstrated in numerous settings (e.g., office work) that paper is a flexible medium with many affordances not supported by current digital systems [8]. Paper-based documents have important qualities for interaction that should be considered in conjunction with the benefits of digital communication tools.

In this work we characterize the affordances of pen and paper interfaces in the setting of speech-language therapy, highlighting their importance and pervasiveness. We analyze how paper-based interfaces are currently combined with speech-based interactions. Based on this, we introduce a prototype that bridges the familiarity of current paper-based low-tech AAC techniques with the dynamic nature of hightech computer devices. We use Livescribe Pulse Smart Pens ${ }^{6}$ as AAC devices and tools to support therapy practices. Finally, we describe how our mixed paper-digital system benefits speech-language therapy.

\section{FIELD RESEARCH}

We conducted five months of field observations and prototype exploration with 15 speech-language therapists (three licensed therapists and 12 therapy students) working with adults during structured speech-language therapy sessions. Field research involved observations of interactions between therapists and clients, during which we recorded detailed field notes about therapy activities, face-to-face interaction, and the material environment. We also conducted regular interviews and prototype reviews. We video recorded therapy sessions and took still photos of the therapy environment. After each session, the therapist debriefed the researcher(s) for five to 30 minutes. This provided a chance

\footnotetext{
${ }^{5}$ http://www. bungalowsoftware.com

${ }^{6}$ http://www.livescribe.com
}

to better understand why certain therapy techniques were used, any additional challenges the client or therapist faced that day, and to answer questions about observations. While speech-language therapists have clients with a range of conditions such as dementia, Lou Gehrig's Disease (ALS), or Parkinson's Disease, our current work focuses on therapy practices with adults who have aphasia and apraxia of speech.

\subsection{Settings}

We observed the practice of speech-language therapy in two settings. The first setting involves ongoing observation of one speech-language therapist (JB) at a continuous care retirement community. Our research team has observed JB on a weekly basis for four months, each week conducting one to three hours of observation. JB has been a licensed and practicing speech-language therapist for four years. He works at this retirement community and at other local senior care facilities. In this setting we observed how the therapist worked with two older adult clients (age 88 and 90) during individual weekly hour-long therapy sessions. We detail the communication abilities and therapy needs of these two clients in Section 6, both of whom had a stroke resulting in expressive aphasia and apraxia of speech. Observations at the retirement community allow our research team to understand the practices of one therapist over time and the progression of therapy techniques as client needs change.

The second setting involves 12 hours of observation at a university-based speech and language clinic that provides services to adults and children with a range of communication challenges. The clinic is part of a graduate program that trains speech-language therapists and provides students with clinical experience. In this setting we observed 12 therapy students working with clients (age 29-59) with aphasia and apraxia of speech resulting from a stroke or traumatic brain injury (TBI). One therapy student worked with each client during one-hour sessions. Some therapy students worked with their clients in a group setting while others engaged in one-on-one structured sessions. Family members and friends were able to observe the therapy sessions from the other side of a one-way mirror. After watching a session, the son of a man undergoing therapy said, "The biggest thing is that he's frustrated. He can understand everything but can't speak." In contrast to long-term observations at the retirement community, this environment exposed the research team to the broad range of communication needs of clients and therapy techniques employed.

\subsection{Observational Themes}

The health condition and communication needs of any individual client are unique, yet field observations reveal several commonalities of speech-language therapy practices. Below we summarize key observations from field research that inform the design of a paper-digital AAC system. Themes were developed based on a detailed review of video data, interviews with therapists, and field notes collected over five months.

\section{Speech-language therapy is collaborative and social}

Language is an inherently multiperson and social phenomenon. The process of speech-language therapy most often involves a therapist working face-to-face with a client at a shared desk or table. Both the therapist and client work collaboratively to reach a mutual understanding during conver- 


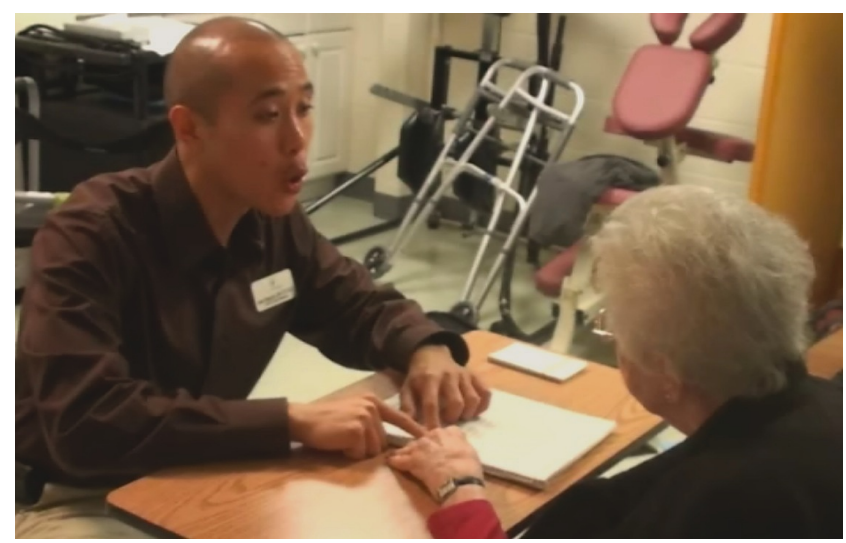

Figure 1: Therapist and client sit face-to-face working on word pronunciation from a paper workbook. Therapist exaggerates as he pronounces the word "join" after the client mistakenly says "jane." All participants consented to the use of photos for publication.

sation, each refining what the other has said until they reach shared agreement. For clients with a severe loss of speech or language, it may take the therapist and client 30 minutes to achieve joint understanding on a single point (e.g., if the client tries to explain what they did over the weekend). On some occasions an individual undergoing therapy will practice language activities on their own as homework, but the majority of structured therapy interaction occurs in a social situation involving two or more people.

\section{The therapy process is multimodal}

Representations in multiple modalities reinforce and reaffirm spoken, written, and auditory forms of language. Figure 1 illustrates multimodal, collaborative interaction between one therapist and client. In this example, the therapist (JB) and client (AF) are both pointing to the word "join" in the workbook. The client reads this word as "jane" so the therapist says "join" and exaggerates his lip movements to emphasize the "o" sound. Therapists present clients with language information in various modalities depending on their communication needs.

\section{Therapy is an ongoing process}

Most therapists meet with their clients on a weekly or biweekly basis. Therapy is ongoing and builds on activities from the prior session. Therapists spend a few minutes at the beginning of each session reminding clients about what they did the previous session and reviewing homework. Since therapy is ongoing, therapists must manage plans and materials for clients over extended periods of time. They often organize paper-based plans in folders and notebooks so that they can document progress.

\section{Sessions are tailored to each client}

Even though several clients may have a similar diagnosis and present in a similar way, therapists must customize activities, goals, and materials for each client. Therapists consider the communication challenges and goals of each client and their individual therapy plan when generating activities for each session. Clients also progress at different speeds and

\footnotetext{
${ }^{7}$ http://www . anoto.com
}

require different forms of stimulation. Furthermore, therapists must be flexible as they address the client's needs from week to week. An emotional change in a client, for example, may render previously successful techniques unproductive.

\section{Practices are rooted in pen and paper interaction}

The structure and nature of speech-language therapy is largely influenced by existing practices with pen and paper. Regardless of a client's condition or communication goals, therapy materials are predominantly paper based. Therapists use paper workbooks, worksheets, communications books, note cards, notepads, and books or novels during each therapy session. Clients whose ability to communicate is severely impaired may write words or draw pictures on blank pieces of paper. Higher functioning individuals may perform paperbased activities such as reading from a word list, reciting minimal pairs (words that sound alike), or writing words to complete sentences. Many therapists also use paper to document treatment plans and notes about a client's progress.

\section{PAPER-DIGITAL PROTOTYPE}

Paper is an important medium for collaboration and shared interaction, and it is pervasive in speech-language therapy. Many affordances of paper play an important role in this setting: paper is inexpensive, flexible, portable, and disposable. Paper materials may be left with a client for home use or filed away by a therapist for documentation. Compared to other high-tech alternatives, pen and paper interaction is familiar and comfortable for most older adults, the predominant population undergoing speech-language therapy.

Existing technology for digital pen and paper $^{7}$ enables static paper documents to be turned into interactive interfaces that can be used to recognize handwritten information, link to digital media such as sound or voice recordings, or trigger specific digital applications on a separate computer either in real time (by streaming the collected information over bluetooth) or in batch-mode (by connecting the digital pen to a computing device at a later time). This technology enables users to exploit rich digital services while keeping the natural interaction common in traditional pen and paper interfaces. The system is based on a special digital pen that integrates a processor, memory, and an infrared camera able to interpret a unique dot-pattern printed on standard paper. By decoding the printed pattern, the device can track the pen's position on paper in real-time.

Digital pen and paper technology has been explored for interaction with a range of digital documents and resources (e.g. text processors [12], PowerPoint presentations [9], field scientist notebooks [13], collaborative annotations [10]), but it has yet to be explored as an assistive technology for adults with communication challenges. The recent introduction of

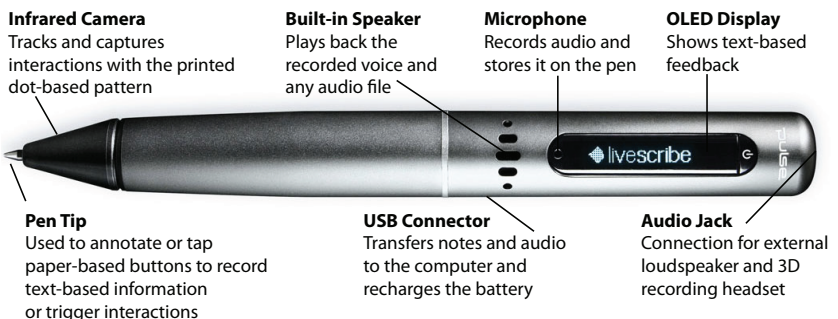

Figure 2: Livescribe Pulse Smart Pen 


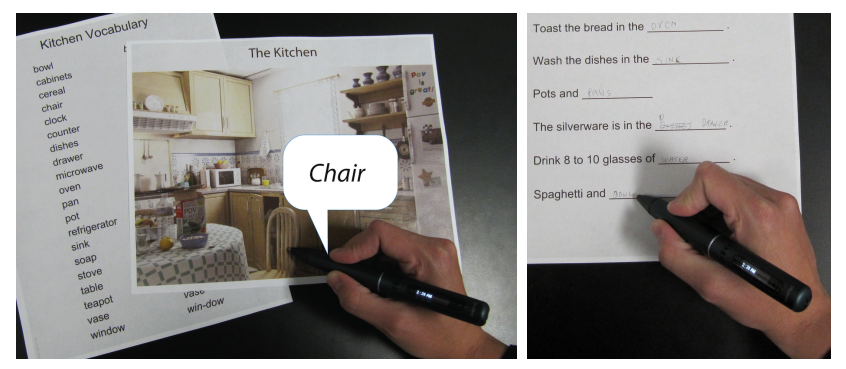

Figure 3: Left: Point-and-playback application, touching on an image or word plays associated audio. Right: Handwriting recognition, handwritten text is stored on the pen and played back.

a novel kind of digital pen, the Livescribe Pulse Smartpen, is particularly promising for speech-language therapy. This pen (shown in Fig. 2) is a "pentop computer" supporting multimodal interactions based on the combination of capturing and recognizing paper-based handwriting with audio recording and playback. In addition to these multimodal capabilities, Livescribe technology enables custom-made applications to be deployed directly on the pen. Field observations indicate that the combination of audio input and output with paper-based interactions may be useful for supporting speech-language therapy. As described earlier, pen and paper interaction is pervasive in current therapy practices, and both multimodality and collaboration are essential components of therapy. Moreover, Livescribe digital pens are relatively inexpensive (under $\$ 200$ ) and readily available.

In order to design, develop, and deploy our custom applications and related interactive paper documents, we exploited the standard Livescribe development toolkit. This tool is an Eclipse plug-in requiring development in Java Micro Edition (J2ME) and is not appropriate for direct use by therapists unless they have programming experience. We developed sample interactive paper prototypes to demonstrate the Livescribe pen's capabilities to therapists. As shown in Figure 3, we produced interactive worksheets by modifying existing paper-based documents used during current therapy sessions. Interactive paper documents cover topics such as naming items in a visual scene display, completing sentences, speaking minimal pair words, listening to the name of a word or icon spoken aloud, and recording one's own speech. In addition, clients may practice writing words and phrases. The pen's handwriting recognition facilities automatically detect keywords and phrases that are written by hand. Practicing speech articulation and constructing sentences verbally is enabled through the pen's audio recording facilities. Clients may record phrases of speech and then play them back for reflection. Central to therapy practices, the system supports multimodal interaction and multimodal representations of language.

\section{PROTOTYPE REVIEW}

As an initial step in the evaluation process, we reviewed the pen and paper prototypes with 15 speech-language therapists, including: JB at the retirement community, the two supervisors of the university clinic, and 12 therapy students. The prototype reviews were conducted in a focus group style to encourage idea generation and open discussion about the positive and negative aspects of this approach.

\section{Appeal of pen and paper interaction}

Therapists thought pen and paper was an appropriate medium for supporting interaction, especially for older clients. The simplicity of pen and paper interaction was key. For one man with aphasia and severe apraxia of speech, a clinic supervisor said, "This would be a great tool for him for a lot of reasons. For one, he's more of a paper and pencil kind of guy, which you saw today, he wanted to draw, draw, draw. He's been resistive to any kind of a device. We've talked to him about devices, we've talked to him about the Lingraphica." The clinic supervisor commented on another client, "[He] has had a lingraphica for over a year, but he hasn't touched it in over nine months... It's hard to navigate through, it's overwhelming. It's too much for a lot of clients." The other clinic supervisor said, "A lot of our clients don't want to use communication books or computers. They may be more inclined to use it if it's still a communication book model but all they have to do is point and it speaks." The simple, familiar, and straight-forward interaction of paper and pen was appealing to therapists given the cognitive and motor challenges many clients face. JB and the other therapists also wanted marking and non-marking pen tips (which Livescribe provides) and to laminate certain pieces of paper to make them hold up longer. The Livescribe pen works with laminated paper and paper behind a slip cover within a binder. Generating ideas for paper-based materials was not a challenge for therapists. A clinic supervisor suggested for one client, "We could have scanned the picture of his children on this dot paper and have the names right there because he obviously knows their names but just couldn't say them."

\section{May reduce communication barriers}

Several therapists thought that the system would allow them to better understand what a client "knows" by reducing the communication barrier. The student working with the man with severe apraxia said, "Even for [him], with something like that [the digital pen] we'd understand more of what he knows rather than assuming or estimating. I think he gets it, but having something more definite would be good." Another student agreed, "It would give you insight into what they really know... Get past that communication barrier."

\section{Custom, human-generated audio}

The ability for the pen to record and replay audio was important to therapists. JB wanted to use the recording facilities to help his clients reflect on how they said certain words. A student in the clinic said, "One of my clients I give multisyllabic words to practice at home for apraxia, but he doesn't have the model of how to say it right, so if he at least had the digital pen]... it would be good." Many AAC devices use a computer synthesized voice, which may not provide clients with an accurate model of how to say words. Another student commented, "The pen puts intonation in there, which is different from a computer voice which doesn't represent it right." She continued, "My client has problems with multisyllabic words. He puts the intonation on the wrong syllable." Recording custom, human-generated audio was an important feature of the system.

Enable independent practice for clients who live alone JB explained that one challenge his clients face is that they often live alone and can become socially isolated. Residents may not have people with whom they regularly interact and 
practice communicating. A similar sentiment was echoed by therapists at the university clinic. One clinic supervisor said, "So many of our clients live alone... I'm thinking home programs for a lot of our clients who live alone, and they don't have a partner or caregiver or someone to help them with their homework. They end up getting frustrated and not doing it." While therapy is a collaborative and social process, supporting independent language activities at home is a positive benefit of the system. The clinic supervisor said to a student, "With your other client...he has this breakdown with phoneme representation... Again this is a gentleman who lives alone, so to have some kind of recording so he can hear. So he can write it and hear it, that would be really good for him."

\section{Time and cost}

Therapists have limited time to prepare for each client, so naturally they were concerned about how much time it would take to prepare the interactive worksheets. Developing a way to help therapists quickly and easily create custom interactive worksheets is a main goal of our future work. For the present study, a researcher was available to generate custom content for therapists using the digital pen with clients.

In addition to time, therapists were concerned about the cost of the device, as this is a central challenge in adopting high-tech AAC devices. When told that the Livescribe pen costs under $\$ 200$, the clinic supervisor said, "I think $\$ 200$ is great, especially compared to an $\$ 8000$ Lingraphica... The pen is very, very affordable, but the software and printer may be a concern." Investing in a digital pen and paper system would require a retirement community or clinic to purchase a high-resolution color printer capable of printing the custom dot pattern. Such a printer may cost $\$ 400-500$ and could be a shared resource. Alternatively, a commercial printing service with only a per page charge could be used.

\section{CASE STUDY EVALUATIONS}

In addition to understanding the perspective of speech-language therapists, we wanted to explore how the pen and paper system might support a therapist working with older adult clients. We created custom therapy materials to support JB working with two clients at the retirement community: $\mathrm{AF}$ and MW. JB integrated the digital pen and interactive paper materials into his therapy plans for these clients. With each client, JB introduced the pen and paper system, demonstrated the desired interaction, and then supported the client as they learned to use the device. JB turned the pen on and off for each client by pressing the power button and started the application by touching the pen to the paper materials. We examined how these two older adults learned to use, responded to, and adapted to the digital pen and paper system as well as JB's impression of the device. To counteract background noise in the retirement community's outpatient therapy room, we provided an external speaker to amplify the pen's volume; however, the pen's integrated speaker is sufficient in other environments such as the university clinic.

\subsection{Case: Language Rehabilitation}

AF (age 88, female) had a stroke in the left side of her brain approximately one year before we began field observations. As a result, she has aphasia and apraxia of speech that make it challenging for her to construct complete or coherent sentences and articulate words. However, AF is fairly high functioning in that she is able to say many words and generally able to communicate her needs. AF lives alone but is well known throughout the retirement community. She often stops to greet other residents and asks "How are you, dear?" Her communication challenges become more evident during structured tasks where she needs to follow instructions or process competing auditory stimuli. She has fully recovered her motor ability in her hands although she uses a walker for balance.

\section{Materials}

Each week during therapy JB provides a range of paperbased activities for AF. They use workbooks, worksheets, note cards, and any books AF is currently reading to practice word articulation and sentence construction. Before her stroke, AF was an excellent and avid cook. Given this, we created a set of interactive worksheets for AF that center around kitchen activities (Fig. 3, left). The worksheets include object identification with a visual scene display of a kitchen, a vocabulary list, sentence completion, following instructions, and written and auditory comprehension.

\section{Initial use}

JB introduced the digital pen and paper to AF, and she figured out the interaction within a couple minutes. Because of her familiarity with one researcher, AF quickly recognized that the pen's voice was that of the researcher. While the pen was tethered to an external speaker, the cord did not get in the way during interaction. However, the therapist had to monitor the connection and ensure that the speaker wire did not come loose.

$\mathrm{AF}$ was able to use the pen to point to various items in the visual scene display, listen to the object's name, and then pronounce it herself. JB asked her to follow this pattern but at times she would continue to touch on objects without practicing saying the name herself. Her ability to point to both small $(1 \mathrm{~cm})$ and large objects with the pen on the visual scene display was excellent. One problem is that she did not know which items in the kitchen scene were active, as many had audio associated with them, but some small items (e.g., a water bottle) did not. AF also had no difficulty using the pen as a writing instrument with the sentence completion and written comprehension activities. She looked at the paper as the pen read a sentence aloud and then filled in the blank or answered the question.

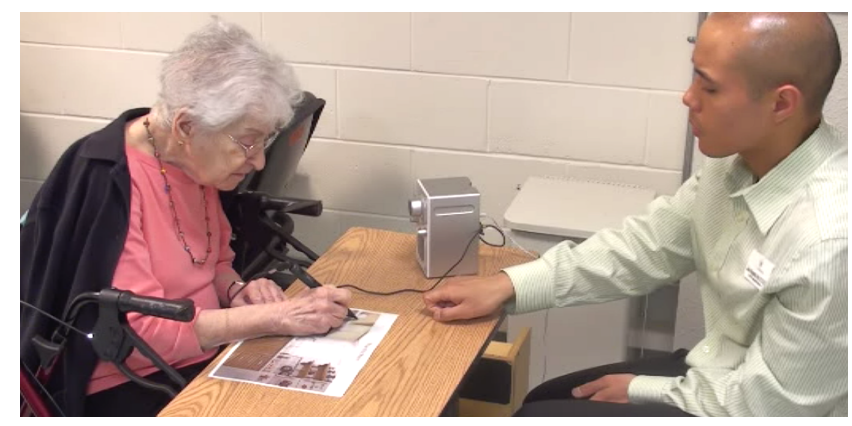

Figure 4: AF uses the digital pen and interactive worksheets to explore a kitchen visual scene display. Tapping on an object plays the name of that object. 


\section{Independent use for homework}

JB continued to use the kitchen activity worksheets during another therapy session with AF. She became more proficient at using the pen. She quickly figured out what she needed to do for each activity and began using the pen with less support from JB. At this point, a goal for AF was to determine whether she would be able to use the pen independently in her home for language practice outside of structured therapy sessions. One researcher brought the digital pen and paper kitchen activities to AF in her home to determine how much support she would need away from JB. While AF remembered using the pen with JB, she needed help turning on the pen and getting started with the first activity. For the homework activities, we modified the existing worksheets to include an answer box that would play the correct answer when tapped, as AF was constantly worried about whether she answered a question correctly. AF was able to independently perform a completing the sentences and a written comprehension task with the digital pen; however, she did not use the pen to listen to words that were difficult for her to pronounce or to verify that she answered each question correctly. Even with prompting from the researcher, the concept of tapping on an answer box to hear the correct answer was too complex for AF. We also explored the audio recording facilities of the digital pen. AF read aloud a passage from a novel she was currently reading, and the researcher helped her record her voice using the digital pen. When asked what she thought about the pen recording and playing back her reading, she said, "I'm not doing very well," indicating that she mispronounced many of the words. She was able to understand that the pen could record her voice and help her reflect on her speech. The ability to record and replay speech from a client working independently is compelling, yet the process needs to be extremely simple with explicit instructions.

\subsection{Case: Augmenting Communication}

Three years ago MW (age 90, female) had a stroke in the left side of her brain. The stroke resulted in aphasia and apraxia of speech. She participated in therapy with JB for several months immediately following her stroke, then took two years off, and recently began therapy again with JB. She is able to understand written and auditory information, but she has extremely limited speaking and writing abilities. The stroke made her unable to speak any words consistently or write legibly by hand. People who interact with MW primarily ask her yes/no questions, to which she responds with a one word answer (e.g., yes or no), head nod, or shoulder shrug. JB explained of MW, "Sometimes she can say full words, other times nothing... There are times when you talk to her where she has one or two word utterances. Talking to her yesterday, I asked her a very direct yes/no question and she said 'no.' That's just the nature of apraxia." MW has been in this communicative state for three years, and according to JB, she "is grateful for the help but very frustrated... Her goal was always rehabilitation. 'Get me back my speech." Recently, JB decided to shift from rehabilitation to compensatory communication strategies.

\section{Materials}

As a communication aid, JB taped a paper picture board with icons and an alphabet board to MW's bedside table. JB has been working with MW to use this as a means of

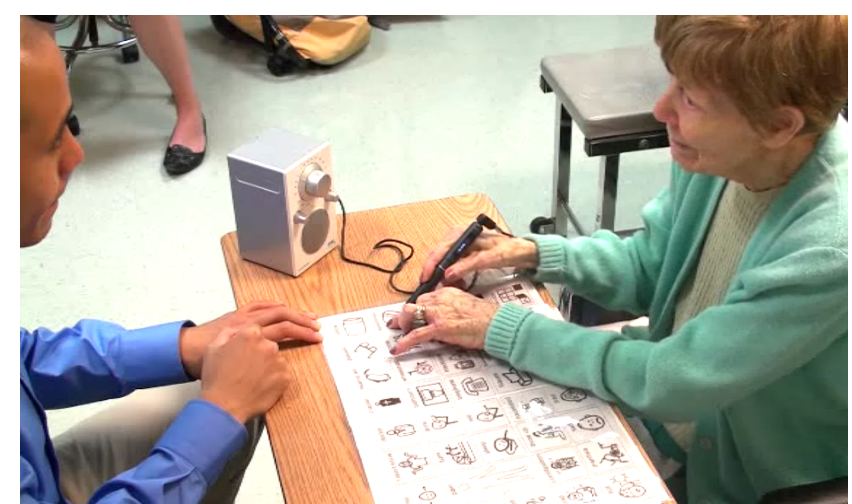

Figure 5: MW tells JB that she is cold and would like a blanket. She points to the cold and then to the blanket icon using the pen and her left index finger.

communication with nurses and community staff (e.g., to request medicine). MW can point (with her index finger) to icons on the picture board to indicate that she is hungry or in pain, for example; however, she often points between two icons or just below an icon, making it difficult for the recipient to understand her. At first, she was slow to scan the picture board and locate an item (took up to 45 seconds to locate a single icon), but with a couple weeks of practice her search speed has increased. We integrated the digital pen with her existing laminated picture boards. Now, touching part of the picture board with the digital pen plays the audio associated with that icon or letter. For this use case, the digital pen had a non-marking tip to serve as a pointing device rather than a writing instrument.

\section{Initial use}

During the first session of use (approximately 45 minutes), MW was able to easily grasp the digital pen in her hand and use it as a pointing device. While holding the pen her fingers did not occlude the camera under the pen tip nor did she accidentally press the power on/off button. At one point during the first session, MW held the pen at an angle where its camera could not read the location on paper. JB helped her adjust the pen position to a more upright angle. MW understood that touching on an icon or letter played audio associated with that item, as she would look up at JB and nod after hearing the sound. Previously, JB taught her to point to icons with her index finger. As a result MW often pointed with her index finger first then the pen or pointed with the pen and her left index finger at the same time. Using the pen while connected to the external speaker was also not a problem.

An interesting segment of activity occurred when JB had MW practice locating icons. She could not find a certain icon after 20 seconds and began to beat her hand on the table. JB then pointed to the frustrated icon and asked, "Are you feeling frustrated?" MW waited for five seconds and pointed with her index finger to the cold icon. JB responded, "You are? Press it." MW touched the cold icon with the pen. JB then said, "What do you want? Using this tell me what you want." MW touched the blanket icon with the pen and her left index finger, then looked up at JB (see Fig. 5). JB responded, "OK, I'll go get you a blanket." He said of the experience, "It was great that she was able to tell me what she wanted." 


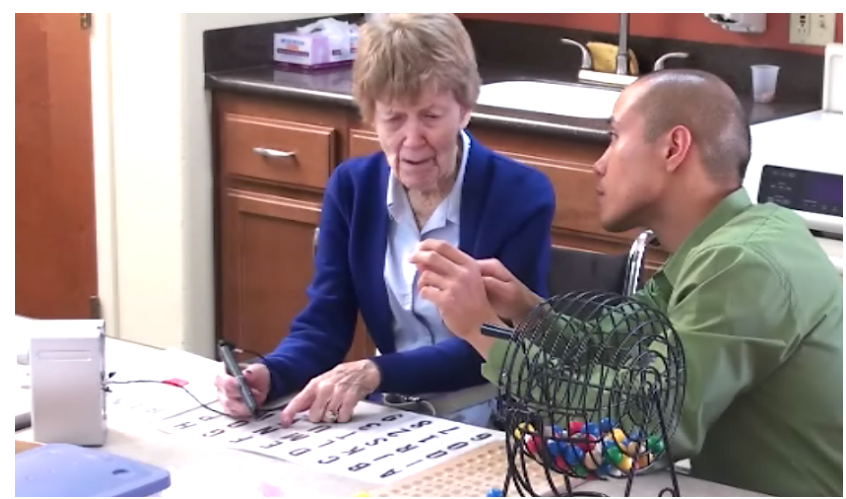

Figure 6: JB helps MW call bingo numbers using her alphabet board and the digital pen.

\section{Applied use for social engagement}

JB spent two additional therapy sessions helping MW learn to use the digital pen and interactive picture boards. The fourth time MW used the digital pen was during a group activity. JB arranged for MW to call out bingo numbers for fellow residents during game time. Applied use of the communication board in this social situation was challenging for MW. This interaction required her to read the bingo letter and number off the ball and then tap the letter and the number for other players to hear. For the first half of the game, JB provided hand over hand guidance to help MW tap out the bingo letter and number. MW was able to do this on her own by the end of the session. She had difficulty, however, with numbers that required double tapping. For example, the number 44, required her to tap 4, release, then tap 4 again. On two different occasions she held the pen down instead of tapping the number twice. The most difficult aspect of interaction for MW was the visual search required to find a letter or number. JB explained afterward, "I think tracking is an issue for her [MW]. It [the picture board] may be too overwhelming. She would look and then give up." Refining the layout, icon size, and icon images for the alphabet and picture boards may help address this problem. This is an easy modification given the flexibility of the digital paper and pen system, and we elaborate this point in the discussion section below. While the task of using the digital pen to call bingo numbers was challenging for MW, the digital pen helped her to engage socially with her peers. She smiled and interacted with other residents, and they cheered for her when she used the pen to call out a bingo number. MW's stroke and subsequent communication loss has made her withdraw socially, so enabling her to engage in this type social setting is a positive benefit.

\section{DISCUSSION}

The multimodal pen and paper interface is a promising tool for speech-language therapy. Our case studies demonstrate that an 88- and 90-year-old were able to successfully use and understand the digital pen and paper system. Conducting an evaluation at the retirement community allowed us to observe a single therapist over a period of time and how two of his clients responded to the technology as an AAC device. One limitation of this approach is that we were constrained to the therapist's caseload of clients, and at the time of this study he only had two clients who were willing and able to participate in research. Exploring multimodal interactive paper technology for a wider age range of clients is one goal of future work. Based on field observations, feedback from therapists, and case studies of use in an authentic setting, we now discuss advantages and challenges of using digital pens in therapy activities.

\section{Usability issues}

Based on our preliminary case study evaluations, the interactive paper materials are fairly easy to use. JB said, "What you have is excellent. It can be incorporated with residents in it's current state." He continued, "It's relatively user friendly, not so much of a high learning curve. It's just using a pen and pointing, then getting feedback. They get an extra notch of stimulation." Several aspects of interaction require further exploration such as whether clients would be able to perform gestures with the pen (e.g., double tap). The form factor of the pen worked well for this audience, but we need to explore the prototype with adults who have less motor ability in their dominant hand. The older adults we observed were able to hold the pen successfully without accidentally covering the infrared camera or turning the pen on/off. Occasionally they would hold the pen at an angle almost parallel to the paper, making the pen unable to read it's current location. Future prototypes should have a feedback mechanism (i.e., audio notification) to help users hold the pen at an appropriate angle.

\section{Logistical challenges}

Fitting with the current workplace practices and logistical challenges of a therapist is critical. One challenge with the current device setup is transporting the equipment to different clients. JB explained, "As a therapist, I want to keep it simple. I don't want to lug a bunch of stuff around... plugging things in, going from room to room. I keep going back to a computer. It can all be compact in one." In contrast, one of the clinic therapists said, "I think it would be great for an everyday AAC device because it is so compact." The amount of equipment a therapist is willing to transport to a client's location may largely depend on how much the device benefits a particular client and whether or not an external speaker is necessary.

\section{System flexibility}

The system is appropriate for use in structured one-on-one therapy sessions, group therapy, independent language practice at home, and as an alternative communication device an individual could take with them throughout their daily activities. JB said, "I can see her $[\mathrm{AF}]$ taking these [worksheets] home with this device to help her. For others, it would be a therapy task... Yeah, it would be great for in home use." With refinement and additional user training, the interactive paper system would be appropriate for a client like AF to use as a homework tool. MW could carry the pen with her throughout the day with a smaller picture board, serving as an alternative communication device.

Customization of activities allows the digital pen to be useful for wide range of individuals with aphasia. The flexibility of paper allows for rapid generation of multiple interfaces that can be tried out with clients. This allows for interface characteristics such as the layout, size, and color scheme to be changed with limited effort. Therapists may also cut out and paste pieces of interactive paper in various ways to rearrange an interface according to a client's needs. 


\section{Device adoption and acceptance}

As with other assistive devices, adoption depends ultimately on what the client will accept. JB said of MW and her husband, "I showed them DynaVox, and her husband was like, 'I don't know if I want a device. I want [her] voice back." JB elaborated, "Acceptance of [a device] is one thing. We must slowly introduce it because this is effectively their voice... It all depends on them ultimately." The therapists at the university clinic suggested that some of their clients who were resistive to more high-tech AAC devices might find the digital pen a suitable alternative.

\section{System for content generation}

Currently a researcher must aid the therapist in generating pen-based content for weekly therapy activities. This was appropriate for the present study where we are just starting to understand the potential of multimodal pen and paper interfaces to support therapy practices. However, given the different needs and requirements of clients, a more flexible approach allowing therapists and potentially family members to define extensible multimodal interfaces is needed. We are developing a system for generating custom digital pen and paper content. We envision a system that enables therapists to define any paper-based interface, exploiting current authoring tools and digital interfaces, and deploy it as interactive paper documents by printing the developed materials on paper augmented with the special dot pattern. The paper-based interface would then serve as a control panel to record custom audio for interaction with clients and associate specific interactions (e.g. handwriting recognition, playback, recording, etc.) to custom areas of the interactive paper sheet. We believe that speech-language therapy would benefit from the flexibility introduced by multimodal pen and paper interfaces. However, if digital pens are to be adopted for long term use in this context, it is necessary to provide a flexible, extensible, and easy-to-use system that enables custom content generation.

\section{CONCLUSION AND FUTURE WORK}

This paper examined the practices of speech-language therapy and how a multimodal digital pen and paper system might support this process. Our analysis presents a first look at the opportunities enabled by this kind of interaction. The two case studies demonstrate the feasibility and appeal of this idea, but the potential for multimodal pen and paper interaction to support therapy processes is seemingly infinite because of the flexible and pervasive nature of paper. Beyond structured therapy activities, an individual could carry a pocket-sized flip book to support daily interaction or print interactive content on stickers or note cards to place in a home or clinic environment. The ability for digital pens to record interaction, both writing and spoken dialogue, make them a promising device for documenting therapy activities over the duration of treatment. Therapists can automatically log client activity and archive notes about client progress to support assessment (similar to the Abaris system [5]). Digital pens may also be combined with computer display systems, and the potential to integrate more dynamic content presented on a multitouch computer with a digital pen is a promising avenue of exploration. In our ongoing work we are examining pen-based technologies in this setting with a larger group of therapists working with younger clients and continuing development on a system for custom content generation. Introducing such a system for generation of multimodal paper-digital interfaces would open up a range of new possibilities and opportunities for therapy.

\section{ACKNOWLEDGMENTS}

This work is funded by NSF grant 0729013. We thank our study participants and Livescribe for donating digital pens.

\section{REFERENCES}

[1] M. Allen, J. McGrenere, and B. Purves. The field evaluation of a mobile digital image communication application designed for people with aphasia. ACM Trans. Access. Comput., 1(1):1-26, 2008.

[2] D. Calder. SpeechKit: a multimedia speech tool. In Proceedings of iiWAS '08, Linz, Austria, November 2008.

[3] S. Chandler, J. Harris, A. Moncrief, and C. Lewis. Naming practice for people with aphasia as a mobile web application. In Proceedings of Assets '09, Pittsburgh, USA, October 2009.

[4] S. Devlin and G. Unthank. Helping aphasic people process online information. In Proceedings of Assets '06, Portland, USA, October 2006.

[5] J. Kientz, S. Boring, G. Abowd, and G. Hayes. Abaris: Evaluating automated capture applied to structured autism interventions. In Proceedings of Ubicomp '05, Tokyo, Japan, September 2005.

[6] K. Moffatt, J. McGrenere, B. Purves, and M. Klawe. The participatory design of a sound and image enhanced daily planner for people with aphasia. In Proceedings of CHI '04, Vienna, Austria, April 2004.

[7] S. Oviatt, P. Cohen, L. Wu, J. Vergo, L. Duncan, B. Suhm, J. Bers, T. Holzman, T. Winograd, J. Landay, J. Larson, and D. Ferro. Designing the user interface for multimodal speech and pen-based gesture applications: state-of-the-art systems and future research directions. Hum.-Comput. Interact., 15(4):263-322, 2000.

[8] A. J. Sellen and R. Harper. The Myth of the Paperless Office. MIT Press, November 2001.

[9] B. Signer and M. C. Norrie. PaperPoint: A Paper-Based Presentation and Interactive Paper Prototyping Tool. In Proceedings of TEI '0\%, Baton Rouge, USA, February 2007.

[10] J. Steimle, O. Brdiczka, and M. Mühlhäuser. CoScribe: Integrating Paper and Digital Documents for Collaborative Kknowledge Work. IEEE Transactions on Learning Technologies, 2(3):174-188, 2009.

[11] K. Tee, K. Moffatt, L. Findlater, E. MacGregor, J. McGrenere, B. Purves, and S. S. Fels. A visual recipe book for persons with language impairments. In Proceedings of CHI '05, Portland, USA, April 2005.

[12] N. Weibel, A. Ispas, B. Signer, and M. C. Norrie. PaperProof: A Paper-Digital Proof-Editing System. In Proceedings of CHI '08, Florence, Italy, April 2008.

[13] R. B. Yeh, C. Liao, S. R. Klemmer, F. Guimbretière, B. Lee, B. Kakaradov, J. Stamberger, and A. Paepcke. ButterflyNet: A Mobile Capture and Access System for Field Biology Research. In Proceedings of CHI '06, Montréal, Canada, April 2006. 